\title{
Observation of the structural phase transition in manganite films by magneto-optical imaging
}

\author{
V.K.Vlasko-Vlasov ${ }^{1,2}, \quad$ Y.Lin ${ }^{1}, \quad$ U.Welp ${ }^{1}, \quad$ G.W.Crabtree', $\quad$ D.J.Miller ${ }^{1}$, \\ V.I.Nikitenko ${ }^{2}$ \\ 'Argonne National Laboratory, Argonne, IL $60439 \quad$. OCT 121993

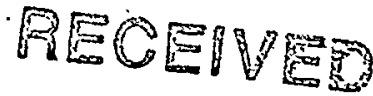 \\ ${ }^{2}$ Institute of Solid State Physics RAS, Chernogolovka, Russia 142432 S
}

A high-resolution magneto-optical imaging technique is used to reveal the formation of twins occurring during a martensitic phase transition at $-105 \mathrm{~K}$ in LCMO films grown on STO substrates. The magnetic contrast arises due to the magneto-elastic tilts of the $\mathrm{Mn}$ - magnetic moments in the twins. Different magnetic structures are found in LCMO films grown on $\mathrm{MgO}, \mathrm{NGO}$, and LAO substrates showing the importance of the substrate material for the manganite film properties.

The submitted manuscript has been created by the University of Chicago as Operator of Argonne National Laboratory ("Argonne") under Contract No. W-31-109-ENG-38 with the U.S. Department of Energy. The U.S. Government retains for itself, and others acting on its behalf, a paid-up, nonexclusive, irrevocable worldwide license in said article to reproduce, prepare derivative works, distribute copies to the public, and perform publicly and display publicly, by or on behalf of the Government. 


\section{DISCLAIMER}

This report was prepared as an account of work sponsored by an agency of the United States Government. Neither the United States Government nor any agency thereof, nor any of their employees, make any warranty, express or implied, or assumes any legal liability or responsibility for the accuracy, completeness, or usefulness of any information, apparatus, product, or process disclosed, or represents that its use would not infringe privately owned rights. Reference herein to any specific commercial product, process, or service by trade name, trademark, manufacturer, or otherwise does not necessarily constitute or imply its endorsement, recommendation, or favoring by the United States Government or any agency thereof. The views and opinions of authors expressed herein do not necessarily state or reflect those of the United States Government or any agency thereof. 


\section{DISCLAIMER}

Portions of this document may be illegible in electronic image products. Images are produced from the best available original document. 


\section{INTRODUCTION}

Manganites are a unique class of materials possessing unusually strong coupling between the three major subsystems: electronic, magnetic and crystallographic ${ }^{1}$. Interest in this system increased strongly after the discovery of the colossal magnetoresistance (CMR) in perovskite manganites ${ }^{2}$ promising wide applications in electronics and magnetic recording. Previous and recent intense studies of bulk perovskites with different substitutions resulted in the construction of phase diagrams showing a rich variety of structural, magnetic, and electronic phases in manganites depending on the composition, temperature and magnetic field ${ }^{3-5}$. Although until today the mechanism of the CMR effect is under debate many physical aspects of these diagrams were clarified and associated with the specific role of $\mathrm{MnO}_{6}$ octahedra which determine magnetic exchange and the electron transfer through the Mn-O-Mn bonds.

Unfortunately, the data for bulk materials can not be directly transferred for the analysis of manganite films showing the CMR effect orders of magnitude larger than in bulk samples. The inevitable mismatch of the film and substrate lattice parameter induces stresses in a film and modifies its properties. One should note that it is very difficult to analyze the lattice structure of a thin film using common structural techniques (X-ray and neutron scattering). Until now not much is known about different phases of manganite films on different substrates.

In the present work we report magneto-optical (MO) observations of the appearance of twins at $\mathrm{T}_{\mathrm{S}} \sim 105 \mathrm{~K}$ in $\mathrm{La}_{1-x} \mathrm{Ca}_{x} \mathrm{MnO}_{3}$ films with $\mathrm{x}=1 / 3$ grown on $\mathrm{SrTiO}_{3}$ (STO) substrates $^{6}$. The formation of pronounced twins manifests the transition from the stretched tetragonal high-temperature phase to the orthorhombic low-temperature (below $\mathrm{T}_{\mathrm{s}}$ ) phase in the film. This transition is triggered by the cubic to tetragonal phase transformation in the STO substrate at nearly the same temperature. The transition in the STO substrate is also accompanied by the formation of twins. However, the twins in the substrate and in the film 
are totally incoherent: they have different size and directions of the domain walls. Therefore, the twinning in the substrate produces an average stress that provides the conditions for the phase transition in the manganite film. This transition does not exist in bulk LCMO and is also not found in films on $\mathrm{NdGaO}_{3}(\mathrm{NGO}), \mathrm{MgO}$, and $\mathrm{LaAlO}_{3}(\mathrm{LAO})$ substrates grown simultaneously with the films on STO.

MO studies of remagnetization processes in LCMO/STO films reveal the inhomogeneous rotation of magnetic moments in the film plane associated with microdomains of differently oriented monoclinic distortions. The dominating effect of these domains on the conductivity of LCMO-films can explain the observed small variation of the resistance of LCMO/STO films at $\mathrm{T}_{\mathrm{S}}$.

In films on NGO and MgO substrates the remagnetization proceeds through the formation and motion of macroscopic magnetic domains with out-of-plane and in-plane magnetic moments. In films on LAO domain structures of the spin-flop type appear in sufficiently strong in-plane fields. The observed variety of magnetic structures and their changes in external fields confirm the crucial effects of the film/substrate lattice mismatch on the physical properties of manganite films.

\section{EXPERIMENT AND RESULTS}

$\mathrm{La}_{2 / 3} \mathrm{Ca}_{1 / 3} \mathrm{MnO}_{3}$ films were grown on (001) STO, MgO, NGO, and LAO substrates in the same course of the pulsed laser deposition. 1000,1200 , and $1500 \AA$ thick films grown in three different runs were deposited at 200 to $800 \mathrm{mTorr}_{2} \mathrm{O}_{2}$ pressure and substrate temperatures of about $750^{\circ} \mathrm{C}$. After deposition the films were annealed for 1 hour in 700 mTorr of $\mathrm{O}_{2}$ at $750^{\circ} \mathrm{C}$. X-ray rocking curves and TEM images confirm the epitaxial film growth. The global characterization of the samples was achieved with measurements of the temperature and field dependence of the magnetization, $\mathrm{M}(\mathrm{H}, \mathrm{T})$, in a SQUID magnetometer and with 4-point resistivity measurements. The local magnetic properties were determined 
formation of twins is a regular feature of martensitic phase transformations ${ }^{8}$. They reduce the overall stresses through a transition into a lower symmetry phase. The transition in the LCMO/STO films proceeds from a high-T tetragonal lattice (which is stretched by the larger lattice parameter of the STO substrate) to the orthorhombic phase that is natural for bulk LCMO in a wide temperature range. It is known that STO has also a transition from the cubic to the tetragonal phase around $\sim 110 \mathrm{~K}^{8}$. Observations through the transparent LCMO film without the MO indicator revealed the appearance of twins in the substrate below $107 \mathrm{~K}$. These twins, however, were aligned at $45^{\circ}$ with respect to the twins in the film and had much larger width. This orientation of twins corresponds to directions of principle [100] and [010] axes in STO which are turned by $45^{\circ}$ with respect to those in LCMO. The dependence of twin width $w$ on the sample thickness $t: w \sim t^{1 / 2}$ explains qualitatively the difference of twin size in the film and the substrate. However, in both film and substrate the twin structure is inhomogeneous (with varying w). Therefore, it is impossible to check the square root dependence. We can only note that at $\mathrm{T}<\mathrm{T}_{\mathrm{S}}$ twins are in general narrower in thinner films.

Until now it is not clear whether the reduction of the a-parameter in STO below the transition at $\sim 107 \mathrm{~K}$ or the relaxation of stresses due to the substrate twinning is the main factor providing conditions for the phase transformation in the LCMO film. In any case the incoherence of twin patterns in the film and substrate shows that twinning in the film is a result of the phase transition in it and not just a replica of the twinning in the substrate.

Estimates show ${ }^{6}$ that the measured magneto-elastic tilts by an angle $\varphi$ of the magnetic moments in the twins should be caused by the $\sigma_{x z}$ components of stress tensor: $\varphi \sim 3 \lambda \sigma_{x z} /\left(\mathrm{K}+2 \pi \mathrm{M}_{\mathrm{s}}^{2}\right)$. Here $\lambda$ and $\mathrm{K}$ are the magnetostriction and anisotropy constants and $M_{S}$ is the saturation magnetization. These out-of-plane shear stresses are not expected for twins in bulk samples and are a specific feature of films. In principle, the average stresses at the transition become rather strong which is revealed by the development of cracks at 
$\mathrm{T}<\mathrm{T}_{\mathrm{s}}$

No sharp peculiarities were revealed at $\sim 105 \mathrm{~K}$ neither in the magnetization nor in resistivity curves. Only a strong reduction of the noise in resistivity was detected below $T_{S}$. This indicates the low- $T$ transport in the films dominated by a mechanism that is only slightly affected by the transition. Observations of the magnetic patterns at the edges of circular holes in the films ${ }^{6}$ showed that the remagnetization proceeds through the incoherent rotation of the magnetic moments in microscopic areas. The average magnetization $\mathbf{M}$ produces characteristic contrast at the edges due to the stray fields at the edge segments perpendicular to $\mathbf{M}$. The absence of such a contrast near the coercivity field implies the absence of aligned magnetic moments on the scale of $<1 \mu \mathrm{m}$. This behavior was explained by inhomogeneities of the samples caused by a possible columnar growth structure and by monoclinically distorted microdomains as revealed by TEM studies ${ }^{910}$ of the LCMO films. Obviously, these imperfections could dominate transport and magnetization in our films masking the effects of the phase transition at $T_{S}$ on $R(T)$ and $M(T)$.

Different microstructures as revealed by magneto-optic patterns appear in LCMO films grown on other substrates. These films do not show the transitions at $\sim 105 \mathrm{~K}$. The magnetic patterns of films on $\mathrm{MgO}$ reveal spots surrounded by bright and dark contrast (Fig.3a). They correspond to areas of larger magnetization surrounded by the background of smaller $\mathrm{M}$, and indicate the phase separation in the film.

Films on NGO show bright domains (lower part of Fig.3b) with magnetic moments tilted out of plane as should be the case of compressed films with positive magnetostriction. In the in-plane field domains with in-plane magnetization (their boundaries in Fig.3b are parallel to the film edge) nucleate and grow at the expense of domains with tilted $\mathbf{M}$.

Films on LAO do not show any magnetic contrast in small fields. But in the inplane field $>0.5 \mathrm{kOe}$ bright and dark stripes appear along the edge (Fig.3c). They can be interpreted as spin-flop domains implying the antiferromagnetic ordering in the LCMO films on LAO. Further study of these magnetic patterns is under way. 
In conclusion, a martensitic phase transition in LCMO/STO films is found through the observation ofa sharp formation of twins at $\sim 105 \mathrm{~K}$. This transformation is triggered by the phase transformation in the substrate at nearly the same temperature which induces changes in strains allowing for the transition in the film to occur. The comparison of magnetic patterns in films on different substrates reveals dramatic effects of the substrate material on the film structure. Studies of these effects are necessary for understanding physics of CMR in manganite films as well as for their applications in microelectronics.

The work was supported by the U.S.DOE, BES-Materials Sciences, under Contract \#W-31-109-ENG-38. 
References

${ }^{1}$ A.J.Millis, Nature 392, 147 (1998).

${ }^{2}$ S.Jin, H.M.O'Bryan, T.H.Tiefel, M.McCormack, W.W.Rhodes, Appl.Phys.Lett. 66, 382 (1995).

${ }^{3}$ E.D.Wollan, W.C.Koehler, Phys.Rev. 100, 545 (1955).

${ }^{4}$ Y.Tokura , Y.Tomioka, H.Kuwahara, A.Asamitsu, Y.Moritomo, M.Kasai, J.Appl.Phys 79, 5288 (1996).

5 P.G.Radaelli, G.Iannone, M Marezio, H.Y.Hwang, S-W.Cheong, J.D.Jorgensen, D.N.Argyriou, Phys.Rev.B 56, 8265 (1997).

6 V.K.Vlasko-Vlasov, Y.Lin, U.Welp, G.W.Crabtree, D.J.Miller, V.I.Nikitenko submitted to Phys.Rev.Lett.

${ }^{7}$ V.K.Vlasko-Vlasov, G.W.Crabtree, U.Welp, V.I.Nikitenko in:"Physics and Materials Science of Vortex States, Flux Pinning and Dynamics", Ed.R.Kossowski et al., Kluwer Ac.Pub., p.205, 1999.

${ }^{8}$ J.A.Krumhansl, in Disorder in Condensed Matter Physics, Ed. J.A.Blackman and J.Taguena, Clarendon Press, Oxford, 1991, p.3.

${ }^{9}$ C.J.Eastell et al., in "Proceedings 1999 American Ceramics Society", Indianopolis, IN, April 1999.

10 O.I.Lebedev, G.Van Tendeloo, S.Amelinckx, B.Leibold, H.-U.Habermeier, Phys.Rev.B 58, 8065 (1998). 


\section{Figure captions}

Fig.1 (a) Field cooled and zero field cooled magnetization curves, $M(T)$, measured in an inplane field of 50 Oe. Hysteresis loops measured in (b) in-plane and (c) normal fields for a $1500 \AA ̊$ thick LCMO film.

Fig.2 MO images of the magnetic patterns in the LCMO/STO film above -(a) and below (b) $105 \mathrm{~K}$. In (a) only stray fields at the film edges (bright and dark lines) and at a $38^{\circ}$ grain boundary (dark line across the sample) are observed. Twin domains are revealed below $\mathrm{T}_{s}$ in (b). The sample width is $2 \mathrm{~mm}$.

Fig.3 Magnetic patterns in LCMO films on MgO - (a), NGO - (b), and LAO - (c) substrates. The arrows point to the edge of film from the outside. The images were taken at $205 \mathrm{~K}, 150 \mathrm{~K}$ and $165 \mathrm{~K}$ in frames (a), (b) and (c), respectively. 

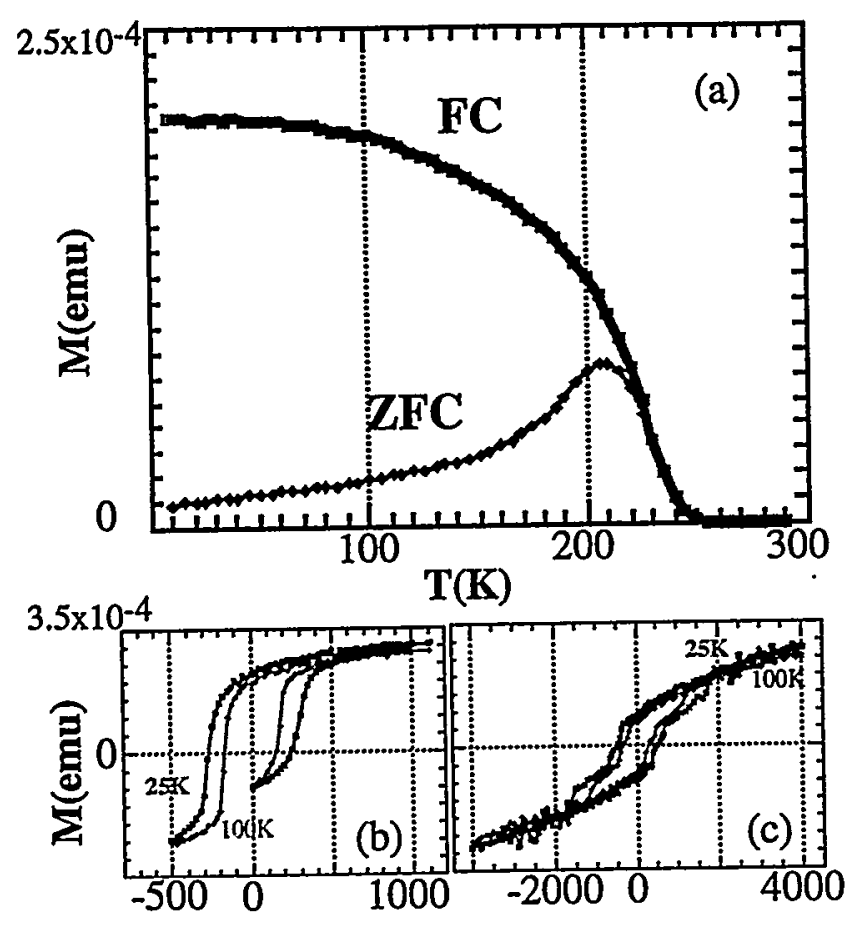

Fig. 1 V.k.Vlask-Vlasov et al., FAP 


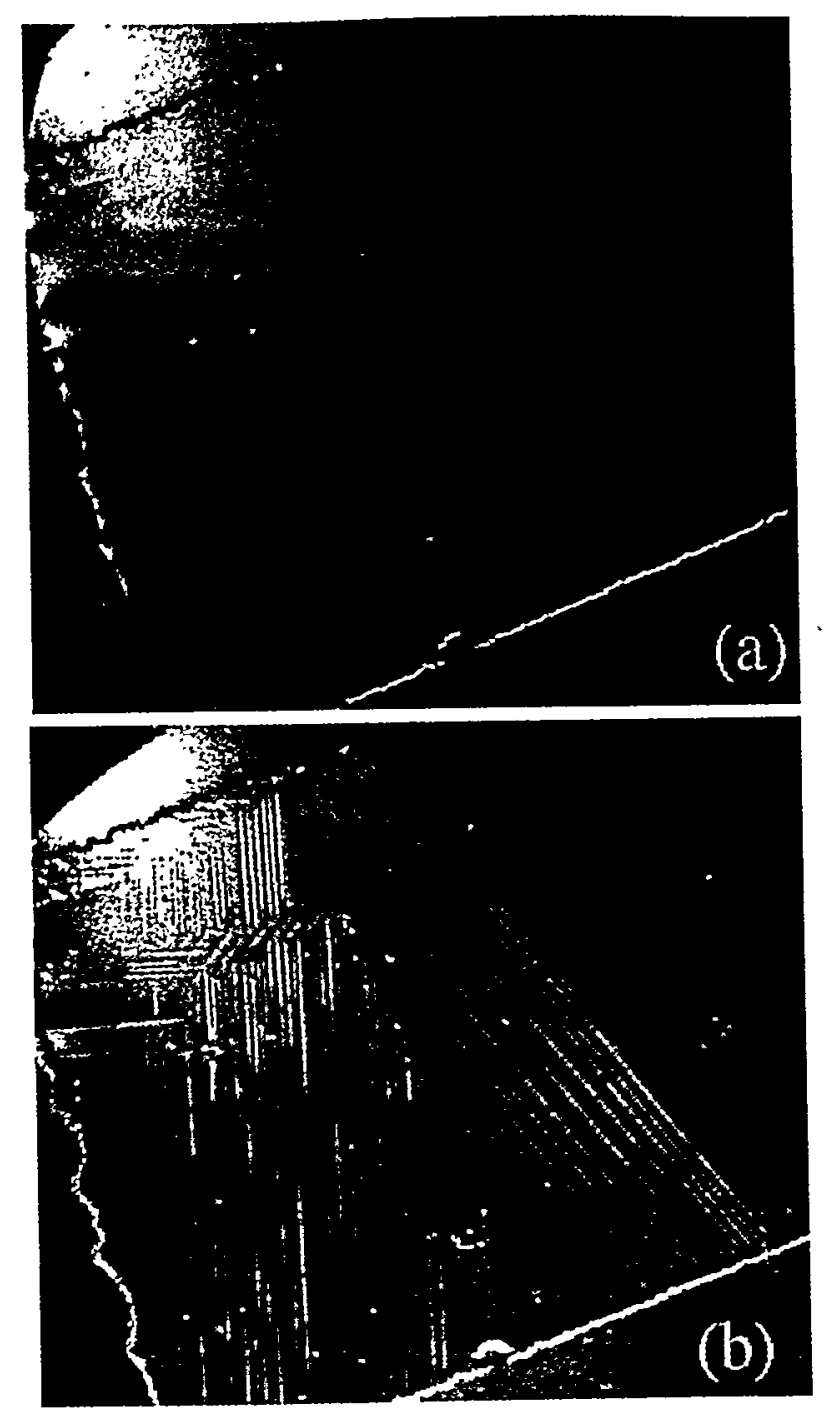

Fig. $\mathcal{L}$ V.K.Vlasko-Vlasov et al., FAP 


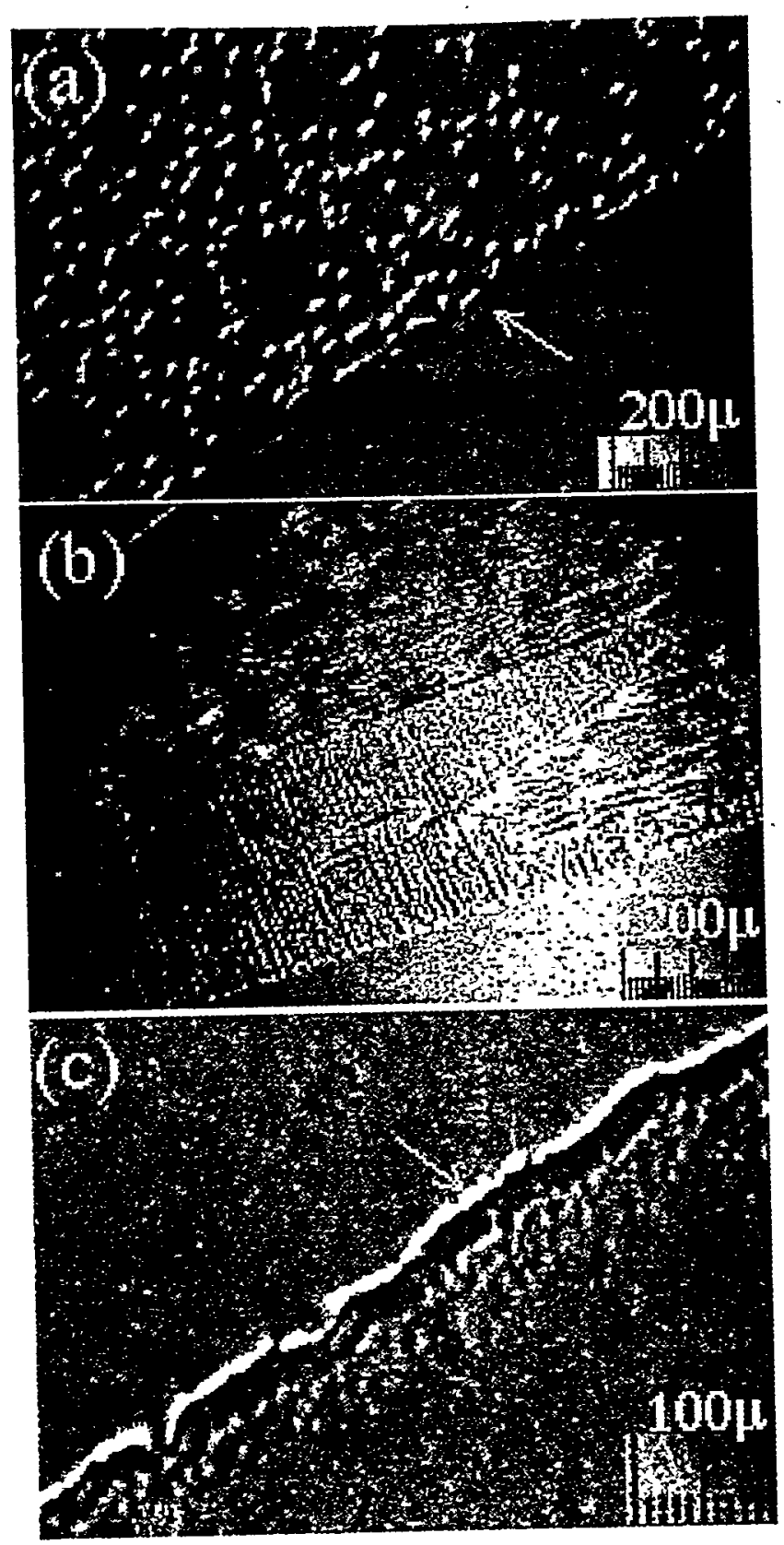

Fig.3 V.K.Vlasko-Vlasev et al., FAP 